\title{
CONVERGENCE RESULTS FOR PROXIMAL POINT ALGORITHM IN COMPLETE CAT(0) SPACE FOR MULTIVALUED MAPPINGS
}

\author{
Samir Dashrutre ${ }^{1}$, Padmavati $^{2}$, Kavita Sakure $^{3}$ \\ ${ }^{1}$ Department of Mathematics, Govt. College, Arjunda, Balod, Chhattisgarh, \\ India, email: samir231973@gmail.com, \\ ${ }^{2}$ Department of Mathematics, Govt. V.Y.T. College, Durg, Chhattisgarh, \\ India, email: padmavati.sudha62@gmail.com \\ ${ }^{3}$ Department of Mathematics, Govt. Digvijay Auto. P.G. College, \\ Rajnandgaon, Chhattisgarh, India email: kavitaaage@gmail.com
}

\begin{abstract}
In this paper, we propose the modified proximal point algorithm with the process for three nearly Lipschitzian asymptotically nonexpansive mappings and multivalued mappings in $\mathrm{CAT}(0)$ space under certain conditions. We prove some convergence theorems for the algorithm which was introduced by Shamshad Hussain et al. [22]. A numerical example is given to illustrate the efficiency of proximal point algorithm for supporting our result.

Key words and Phrases: CAT(0) spaces, Nearly Lipschitzian mappings, Proximal point algorithm, $\Delta$-convergence.
\end{abstract}

\section{INTRODUCTION}

The proximal point algorithm (PPA) is a method for finding a minimizers of convex lower semicontinuous (lsc) function defined on Hilbert spaces was initiated by Martinet [29] in 1970. The PPA has since become extremely popular among the various researchers inclination in the theory of optimization and also exposed many challenging mathematical problems. The rich literature on this subject has become too extensive (see e.g. [7-9,12,13,17,18,21,24,25,36-38]). In particular, the PPA was studied in the framework of Riemannian manifold [10,20], in Hadamard manifold $[4-6,11,27,41]$ and in $\operatorname{CAT}(0)$ space $[8,14-16,34,40]$.

On the otherhand, Markin [28] and Nadler [31] introduced the study of fixed points for multivalued contractions and nonexpansive mappings using the Hausdorff

2020 Mathematics Subject Classification: 47H09, 47H10, 47J25, 65K10.

Received: 24-01-2021, accepted: 23-12-2020. 
metric. Shimizu et al. [39] proved the existence of fixed points for multivalued nonexpansive mappings in convex metric space was established by Shimizu et al. [39], i.e. he proved that every multivalued mapping $\mathcal{T}: Y \rightarrow \mathcal{C}(Y)$ has a fixed point in a bounded, complete and uniformly convex metric space $(Y, d)$, where $\mathcal{C}(Y)$ is family of all compact subsets of $Y$. In this direction to generalize the nonlinear multivalued mappings, Kim et al. [26] introduced the nearly Lipschitzian multivalued mapping.

In 2019, Hussain et al. [22] has been introduced modified proximal point algorithm in complete $\mathrm{CAT}(0)$ space $(Y, d)$ as follows : suppose that $h$ is a convex, proper and lower semi-continuous function on $Y$. The modified proximal point algorithm is given by for $s_{1} \in Y$ and $\pi_{m}>0$

$$
\begin{cases}p_{m}=\operatorname{argmin}_{r \in Y}\left\{h(r)+\frac{1}{2 \pi_{m}} d^{2}\left(r, s_{m}\right)\right\} & \\ q_{m}=\left(1-\gamma_{m}\right) p_{m} \oplus \gamma_{m} A^{m} z_{m}, & \\ r_{m}=\left(1-\beta_{m}\right) q_{m} \oplus \beta_{m} B^{m} y_{m}, & \forall m \in N .\end{cases}
$$

where $z_{m} \in P_{\mathcal{T}}\left(p_{m}\right), y_{m} \in P_{\mathcal{T}}\left(q_{m}\right)$ and $x_{m} \in P_{\mathcal{T}}\left(r_{m}\right)$ for each $m \in N$. Let $\left\{a_{m}\right\},\left\{b_{m}\right\}$ and $\left\{c_{m}\right\}$ be a sequence in $[0,1]$ for all $m \in N$ and $\left\{\pi_{m}\right\}$ be a sequence with $\pi_{m}>0$ for all $m \in N$ and established some $\Delta$-convergence theorems of the proposed algorithm to common fixed points of nonexpansive mappings including a total asymptotically nonexpansive mapping, multivalued mapping and minimizer of a convex function.

In the view of above literature, we propose the modified proximal point algorithm with the process for three nearly Lipschitzian asymptotically nonexpansive mappings and multivalued mappings in $\mathrm{CAT}(0)$ space under certain conditions. We prove $\Delta$ - convergence, strong and weak convergence results for the algorithm which was defined in (1) by Shamshad Hussain et al. [22]. A numerical example is given to illustrate the efficiency of proximal point algorithm for supporting our result.

\section{Preliminaries}

Throughout in this paper, we assume that

$$
G(T)=\{x \in \mathcal{W}: T x=x\}
$$

denote the set of fixed point where $\mathcal{W}$ is subset of $\operatorname{CAT}(0)$ space $(Y, d)$ and $\mathcal{T}$ : $\mathcal{W} \rightarrow \mathcal{W}$ is a mapping. A metric space $(Y, d)$ is called a $\operatorname{CAT}(0)$ space if it is geodesically connected and every geodesic triangle in $Y$ is atleast as thin as its comparison triangle in the Euclidean plane.

A subset $\mathcal{W}$ of a $\operatorname{CAT}(0)$ space $Y$ is said to be convex, if for any $s, r \in \mathcal{W}$, we have $[s, r] \subset \mathcal{W}$, where

$$
[s, r]:=\{t s \oplus(1-t) r: 0 \leq t \leq 1\}
$$


is unique geodesic joining $s$ and $r$. In this paper, we can write $t s \oplus(1-t) r$ for the unique point $q$ in the geodesic segment joining $s$ to $r$ such that

$$
d(s, q)=t d(s, r), \quad d(r, q)=(1-t) d(s, r)
$$

where $t \in[0,1]$.

Definition 2.1. Let $\left\{s_{m}\right\}$ be bounded sequence in a $\operatorname{CAT}(0)$ space $(Y, d)$. For any $s \in Y$, we put

$$
\hat{r}\left(s,\left\{s_{m}\right\}\right)=\lim _{m \rightarrow \infty} \sup d\left(s, s_{m}\right) .
$$

Then,

1. The asymptotic radius of $\hat{r}\left(\left\{s_{m}\right\}\right)$ of $\left\{s_{m}\right\}$ is given by

$$
\hat{r}\left(\left\{s_{m}\right\}\right)=\inf \left\{\hat{r}\left(s,\left\{s_{m}\right\}\right): s \in Y\right\} .
$$

2. The asymptotic center $\mathcal{A}\left(\left\{s_{m}\right\}\right)$ of $\left\{s_{m}\right\}$ is the set

$$
\mathcal{A}\left(\left\{s_{m}\right\}\right)=\left\{s \in Y: \hat{r}\left(\left\{s, s_{m}\right\}\right)=\hat{r}\left(\left\{s_{m}\right\}\right)\right\} .
$$

In complete $\operatorname{CAT}(0)$ space, $\mathcal{A}\left(\left\{s_{m}\right\}\right)$ consists of exactly one point [15].

Definition 2.2. A sequence $\left\{s_{m}\right\}$ in a $\operatorname{CAT}(0)$ space $(Y, d)$ is said to be $\Delta$-convergence to a point $s \in Y$, if $s$ is a unique asymptotic center of $\left\{u_{m}\right\}$ for every subsequence $\left\{u_{m}\right\}$ of $\left\{s_{m}\right\}$. In this case, we write $\Delta \lim _{m \rightarrow \infty} s_{m}=s$ of $\left\{s_{m}\right\}$ and denote $\mathcal{W}_{\triangle}\left(s_{m}\right):=\cup \mathcal{A}\left(\left\{u_{m}\right\}\right)$, where the union is sum over all subsequences $\left\{u_{m}\right\}$ of $\left\{s_{m}\right\}$.

Lemma 2.3. Let $Y$ be a geodesic space in $\mathrm{CAT}(0)$ space. For all $s, r, q \in Y$ and $t \in[0,1]$, we have

(i) $d^{2}((1-t) s \oplus t r, q) \leq(1-t) d^{2}(s, q)+t d^{2}(r, q)-t(1-t) d^{2}(s, r)$;

(ii) $d((1-t) s \oplus t r, q) \leq(1-t) d(s, q)+t d(r, q)$.

Lemma 2.4. ([15]) If $\left\{s_{m}\right\}$ is a bounded sequence in a complete CAT(0) space with $\mathcal{A}\left(\left\{s_{m}\right\}\right)=\{s\},\left\{u_{m}\right\}$ is subspace of $\left\{s_{m}\right\}$ with $\mathcal{A}\left(\left\{u_{m}\right\}\right)=\{u\}$, and the sequence $\left\{d\left(s_{m}, u\right)\right\}$ converges, then $s=u$.

Lemma 2.5. ( [2]) Assume that a subset of a complete $\operatorname{CAT}(0)$ space $(Y, d)$ is closed, convex and $\mathcal{T}: \mathcal{W} \rightarrow \mathcal{W}$ is nearly Lipschitzian mapping. Let $\left\{s_{m}\right\}$ be a bounded sequence in $\mathcal{W}$ such that $\Delta \lim _{m \rightarrow \infty} s_{m}=t$ and $\lim _{m \rightarrow \infty} d\left(s_{m}, \mathcal{T} s_{m}\right)=0$. Then $\mathcal{T} t=t$.

Let $\mathcal{C B}(\mathcal{W})$ be a collection of all nonempty and closed bounded subsets and $\mathcal{P}(\mathcal{W})$ be a collection of all nonempty proximal bounded and closed subsets of $\mathcal{W}$, respectively. Let $\mathcal{H}(.,$.$) be the Hausdorff distance on \mathcal{C B}(\mathcal{W})$ defined by

$$
\mathcal{H}(\mathcal{A}, \mathcal{B})=\max \left\{\sup _{s \in \mathcal{A}} \operatorname{dist}(s, \mathcal{B}), \sup _{r \in \mathcal{B}} \operatorname{dist}(r, \mathcal{A})\right\} \quad \forall \mathcal{A}, \mathcal{B} \in \mathcal{C B}(\mathcal{W}) .
$$

A subset $\mathcal{W} \subset Y \neq \phi$ is said to be proximal if for each $s \in Y$, there exists an element $r \in \mathcal{W}$ such that

$$
d(s, r)=\operatorname{dist}(s, \mathcal{W})=\inf \{d(s, q): q \in \mathcal{W}\} .
$$


It is well known that each weakly compact convex subset of a Banach space is proximal as well as each closed convex subset of a uniformly convex Banach space is also proximal. Many authors have been discussed fixed point in CAT(0) space (see $[1,19,32])$.

Let $\mathcal{T}: Y \rightarrow 2^{Y}$ be a multivalued mapping. An element $s \in Y$ is said to fixed point of $\mathcal{T}$ if $s \in \mathcal{T} s$.

Definition 2.6. A multivalued mapping $\mathcal{T}: Y \rightarrow \mathcal{C B}(Y)$ is called nonexpansive, if for $x, y \in Y$ and for $m \in N$, we have

$$
\mathcal{H}\left(\mathcal{T}^{m} s, \mathcal{T}^{m} r\right) \leq d(\mathcal{T} s, \mathcal{T} r), \quad \forall s, r \in Y .
$$

Definition 2.7. [26] A multivalued mapping $\mathcal{T}: Y \rightarrow \mathcal{C B}(Y)$ is called nearly Lipschitzian with respect to sequence $\left\{v_{m}\right\}$, if for $x, y \in Y$ and for $m \in N$, there exists a constant $k_{m} \geq 0$, such that

$$
\mathcal{H}\left(\mathcal{T}^{m} s, \mathcal{T}^{m} r\right) \leq k_{m}\left(d(\mathcal{T} s, \mathcal{T} r)+v_{m}\right), \quad \forall s, r \in Y .
$$

where the sequence $\left\{v_{m}\right\}$ in $[0, \infty)$ such that $\lim _{m \rightarrow \infty} v_{m}=0$. The infimum of constants $k_{m}$ in (3) is called the nearly Lipschitzian constant of $\mathcal{T}^{m}$, denoted by $\eta\left(\mathcal{T}^{m}\right)$.

A multivalued nearly Lipschitzian mapping $\mathcal{T}$ with sequence $\left(v_{m}, \eta\left(\mathcal{T}^{m}\right)\right)$ is said to be

(1) multivalued nearly nonexpansive, if $\eta\left(\mathcal{T}^{m}\right)=1$ for all $m \in N$,

(2) multivalued nearly asymptotically nonexpansive, if $\eta\left(\mathcal{T}^{m}\right) \geq 1$ for all $m \in$ $N$ and $\lim _{m \rightarrow \infty} \eta\left(\mathcal{T}^{m}\right)=1$,

(3) multivalued nearly uniformly k-Lipschitzian, if $\eta\left(\mathcal{T}^{m}\right) \leq k$ for all $m \in N$,

(4) multivalued nearly uniformly k-contractive, if $\eta\left(\mathcal{T}^{m}\right) \leq k<1$ for all $m \in N$.

The following example of nearly Lipschitzian mapping given by Abbas et al. [2] as follows.

Example 2.8. Assume that $A:(0, \infty) \rightarrow(0, \infty)$ is defined by

$$
A(s)= \begin{cases}1+(s)^{\frac{1}{2}} & s \in(0,1] \\ 2 & s \in(1, \infty)\end{cases}
$$

Similarly, we define here two nearly Lipschitzian mappings in our next two examples as follows.

Example 2.9. Assume that $B:(0, \infty) \rightarrow(0, \infty)$ is defined by

$$
B(s)= \begin{cases}1+(s)^{\frac{1}{3}} & s \in(0,1] \\ 2 & s \in(1, \infty)\end{cases}
$$

Here

Thus

$$
B^{m} x=B^{m} y=2 \quad \forall m \geq 2 .
$$

$$
d\left(B^{m} x, B^{m} y\right) \leq k_{m}\left(d(x, y)+v_{m}\right) \quad \forall m \geq 2
$$

is true for $k_{m} \geq 0$ and for any sequence $\left\{v_{m}\right\}$ in $[0, \infty)$ with $v_{m} \rightarrow 0$. 
Example 2.10. Assume that $C:(0, \infty) \rightarrow(0, \infty)$ is defined by

$$
C(s)= \begin{cases}1+(s)^{\frac{1}{4}} & s \in(0,1] \\ 2 & s \in(1, \infty)\end{cases}
$$

Here

$$
C^{m} x=C^{m} y=2 \quad \forall m \geq 2 .
$$

Thus

$$
d\left(C^{m} x, C^{m} y\right) \leq k_{m}\left(d(x, y)+v_{m}\right) \quad \forall m \geq 2
$$

is true for $k_{m} \geq 0$ and for any sequence $\left\{v_{m}\right\}$ in $[0, \infty)$ with $v_{m} \rightarrow 0$.

Recall that a function $h: \mathcal{W} \rightarrow(-\infty, \infty]$ is convex, if for any geodesic $[s, r]=$ $\left\{c_{s, r}(a): 0 \leq a \leq 1\right\}=\{a s \oplus(1-a) r: 0 \leq a \leq 1\}$ joining $s, r \in \mathcal{W}$, the function $h \circ c$ is convex, i.e.,

$$
h\left(c_{s, r}(a)\right)=h(a s \oplus(1-a) r) \leq a h(s)+(1-a) h(r) .
$$

The Moreau-Yoshida resolvent of function $h$ in the CAT(0) space is given by

$$
\mathcal{J}_{\pi}(x)=\operatorname{argmin}_{r \in Y}\left[h(r)+\frac{1}{2 \pi} d^{2}(r, s)\right]
$$

for any $\pi>0$ and for all $s \in Y$.

Remark 2.11. (1) The resolvent $\mathcal{J}_{\pi}$ of function $h$ is nonexpansive for all $\pi>$ 0 (see [23]).

(2) If $h$ is convex, proper and lower semi-continuous function, then the set of fixed point of the resolvent associated with $h$ coincides with the set of minimizers of $h$ (see [7]).

Lemma 2.12. (see [23,30]) Suppose that $(Y, d)$ is a CAT(0) space. Then for each $r, s \in Y$ and $\pi>0$

$$
\frac{1}{2 \pi} d^{2}\left(\mathcal{J}_{\pi} s, r\right)-\frac{1}{2 \pi} d^{2}(s, r)+\frac{1}{2 \pi} d^{2}\left(\mathcal{J}_{\pi} s, s\right) \leq h(r)-h\left(\mathcal{J}_{\pi} s\right) .
$$

Lemma 2.13. ([3]) Suppose that $(Y, d)$ is a CAT(0) space. Then for each $s \in Y$ and $\pi>\mu>0$

$$
\mathcal{J}_{\pi} s=\mathcal{J}_{\mu}\left(\frac{\pi-\mu}{\pi} \mathcal{J}_{\pi} s \oplus \frac{\mu}{\pi} s\right)
$$

Lemma 2.14. ([39]) Let $\left\{a_{m}\right\},\left\{b_{m}\right\}$ and $\left\{c_{m}\right\}$ be sequences of nonnegative real numbers such that

$$
a_{m+1} \leq\left(1+b_{m}\right) a_{m}+c_{m} \quad \forall m \in N .
$$

If $\sum_{m=1}^{\infty} b_{m}<\infty$ and $\sum_{m=1}^{\infty} c_{m}<\infty$, then $\lim _{m \rightarrow \infty} a_{m}$ exists. 


\section{Main Results}

Theorem 3.1. Let $(Y, d)$ be a complete $\mathrm{CAT}(0)$ space and $\mathcal{W}$ be a nonempty closed convex subset of $Y$. Let $\mathcal{T}: \mathcal{W} \rightarrow P(\mathcal{W})$ be multivalued mapping and $P_{\mathcal{T}}$ be a nonexpansive mapping. Let $h: Y \rightarrow(-\infty, \infty]$ be a proper convex and lower semicontinuous function and $A, B, C: \mathcal{W} \rightarrow \mathcal{W}$ be three nearly Lipschitzian mappings with $\left\{k_{m}\right\},\left\{v_{m}\right\}$ being nonnegative real sequences such that $\sum_{m=1}^{\infty} k_{m}<\infty$ and $\sum_{m=1}^{\infty} \nu_{m}<\infty$. Let $\mathcal{E}:=G(A) \cap G(B) \cap G(C) \cap G(\mathcal{T}) \cap \operatorname{argmin}_{r \in W} h(r) \neq \phi$. Let $\left\{s_{m}\right\}$ be defined by

$$
\begin{cases}p_{m}=\operatorname{argmin}_{r \in Y}\left\{h(r)+\frac{1}{2 \pi_{m}} d^{2}\left(r, x_{m}\right)\right\} & \\ q_{m}=\left(1-c_{m}\right) p_{m} \oplus c_{m} A^{m} z_{m} & \\ r_{m}=\left(1-b_{m}\right) B^{m} y_{m} \oplus b_{m} q_{m} & m \in N \\ s_{m+1}=\left(1-a_{m}\right) r_{m}+a_{m} C^{m} x_{m} & m \in\end{cases}
$$

where $z_{m} \in P_{\mathcal{T}}\left(p_{m}\right), y_{m} \in P_{\mathcal{T}}\left(q_{m}\right)$ and $x_{m} \in P_{\mathcal{T}}\left(r_{m}\right)$ for each $m \in N$. Let $\left\{a_{m}\right\},\left\{b_{m}\right\}$ and $\left\{c_{m}\right\}$ be sequences in $[0,1]$ for all $m \in N$ and $\left\{\pi_{m}\right\}$ be a sequence with $\pi_{m}>0$ for all $m \in N$. Then $\lim _{m \rightarrow \infty} d\left(s_{m}, t\right)$ exists for all $t \in \mathcal{E}$.

Proof. Since $\mathcal{E} \neq \phi$. So we can assume that $t \in \mathcal{E}$ which implies that $t=A t=B t=$ $C t$ and $h(t) \leq h(r)$ for any $r \in \mathcal{W}$. Thus, we have

$$
h(t)+\frac{1}{2 \pi_{m}} d^{2}(t, t) \leq h(r)+\frac{1}{2 \pi_{m}} d^{2}(r, t)
$$

for each $r \in W$, and we have $t=\mathcal{J}_{\pi_{m}} t$ for each $m \in N$. Since $p_{m}=\mathcal{J}_{\pi_{m}} s_{m}$ and $\mathcal{J}_{\pi_{m}}$ is nonexpansive, so we have

$$
d\left(p_{m}, t\right)=d\left(\mathcal{J}_{\pi_{m}} s_{m}, \mathcal{J}_{\pi_{m}} t\right) \leq d\left(s_{m}, t\right)
$$

Now using (4), (5) and Lemma 2.3, we have

$$
\begin{aligned}
d\left(q_{m}, t\right) & =d\left(\left(1-c_{m}\right) p_{m} \oplus c_{m} A^{m} z_{m}, t\right) \\
& \leq\left(1-c_{m}\right) d\left(p_{m}, t\right)+c_{m} d\left(A^{m} z_{m}, t\right) \\
& \leq\left(1-c_{m}\right) d\left(p_{m}, t\right)+c_{m}\left(k_{m}\left(d\left(z_{m}, t\right)+v_{m}\right)\right) \\
& =\left(1-c_{m}\right) d\left(p_{m}, t\right)+c_{m} k_{m} d\left(z_{m}, t\right)+c_{m} k_{m} v_{m} \\
& \leq\left(1-c_{m}\right) \operatorname{dist}\left(p_{m}, P_{\mathcal{T}}(t)\right)+c_{m} k_{m} \operatorname{dist}\left(z_{m}, P_{\mathcal{T}}(t)\right)+c_{m} k_{m} v_{m} \\
& \leq\left(1-c_{m}\right) \mathcal{H}\left(P_{\mathcal{T}}\left(p_{m}\right), P_{\mathcal{T}}(t)\right)+c_{m} k_{m} \mathcal{H}\left(P_{\mathcal{T}}\left(z_{m}\right), P_{\mathcal{T}}(t)\right)+c_{m} k_{m} v_{m} \\
& \leq\left(1-c_{m}\right) d\left(p_{m}, t\right)+c_{m} k_{m} d\left(p_{m}, t\right)+c_{m} k_{m} v_{m} \\
& =\left(1-c_{m}\left(1-k_{m}\right)\right) d\left(p_{m}, t\right)+c_{m} k_{m} v_{m} \\
& \leq\left(1-c_{m}\left(1-k_{m}\right)\right) d\left(s_{m}, t\right)+c_{m} k_{m} v_{m} \\
d\left(q_{m}, t\right) & \leq \mathcal{C} d\left(s_{m}, t\right)+\mathcal{G}
\end{aligned}
$$


where $\mathcal{C}=1-c_{m}\left(1-k_{m}\right)$ and $\mathcal{G}=c_{m} k_{m} v_{m}$.

Now using (4), (6) and Lemma 2.3, we have

$$
\begin{aligned}
& d\left(r_{m}, t\right)=d\left(\left(1-b_{m}\right) B^{m} y_{m} \oplus b_{m} q_{m}, t\right) \\
& \leq\left(1-b_{m}\right) d\left(B^{m} y_{m}, t\right)+b_{m} d\left(q_{m}, t\right) \\
& \leq\left(1-b_{m}\right)\left(k_{m}\left(d\left(y_{m}, t\right)+v_{m}\right)\right)+b_{m} d\left(q_{m}, t\right) \\
& =\left(1-b_{m}\right) k_{m} d\left(y_{m}, t\right)+\left(1-b_{m}\right) k_{m} v_{m}+b_{m} d\left(q_{m}, t\right) \\
& \leq\left(1-b_{m}\right) k_{m} \operatorname{dist}\left(y_{m}, P_{\mathcal{T}}(t)\right)+\left(1-b_{m}\right) k_{m} v_{m}+b_{m} \operatorname{dist}\left(q_{m}, P_{\mathcal{T}}(t)\right) \\
& \leq\left(1-b_{m}\right) k_{m} \mathcal{H}\left(P_{\mathcal{T}}\left(y_{m}\right), P_{\mathcal{T}}(t)\right)+\left(1-b_{m}\right) k_{m} v_{m}+b_{m} \mathcal{H}\left(\mathcal{T}\left(q_{m}\right), P_{\mathcal{T}}(t)\right) \\
& \leq\left(1-b_{m}\right) k_{m} d\left(q_{m}, t\right)+\left(1-b_{m}\right) k_{m} v_{m}+b_{m} d\left(q_{m}, t\right) \\
& \leq\left(1-b_{m}\left(1-k_{m}\right)\right) d\left(q_{m}, t\right)+\left(1-b_{m}\right) k_{m} v_{m} \\
& \leq\left(1-b_{m}\left(1-k_{m}\right)\right)\left(\mathcal{C} d\left(s_{m}, t\right)+\mathcal{G}\right)+\left(1-b_{m}\right) k_{m} v_{m} \\
& d\left(r_{m}, t\right) \leq \mathcal{B C} d\left(s_{m}, t\right)+\mathcal{B G}+\mathcal{F}
\end{aligned}
$$

where $\mathcal{B}=1-b_{m}\left(1-k_{m}\right)$ and $\mathcal{F}=\left(1-b_{m}\right) k_{m} v_{m}$.

Now using (4), (7) and Lemma 2.3, we have

$$
\begin{aligned}
& d\left(s_{m+1}, t\right)=d\left(\left(1-a_{m}\right) r_{m} \oplus a_{m} C^{m} x_{m}, t\right) \\
& \leq\left(1-a_{m}\right) d\left(r_{m}, t\right)+a_{m} d\left(C^{m} x_{m}, t\right) \\
& \leq\left(1-a_{m}\right) d\left(r_{m}, t\right)+a_{m}\left(k_{m}\left(d\left(x_{m}, t\right)+v_{m}\right)\right) \\
& =\left(1-a_{m}\right) d\left(r_{m}, t\right)+a_{m} k_{m} d\left(x_{m}, t\right)+a_{m} k_{m} v_{m} \\
& \leq\left(1-a_{m}\right) \operatorname{dist}\left(r_{m}, P_{\mathcal{T}}(t)\right)+a_{m} k_{m} \operatorname{dist}\left(x_{m}, P_{\mathcal{T}}(t)\right)+a_{m} k_{m} v_{m} \\
& \leq\left(1-a_{m}\right) \mathcal{H}\left(P_{\mathcal{T}}\left(r_{m}\right), P_{\mathcal{T}}(t)\right)+a_{m} k_{m} \mathcal{H}\left(P_{\mathcal{T}}\left(x_{m}\right), P_{\mathcal{T}}(t)\right)+a_{m} k_{m} v_{m} \\
& \leq\left(1-a_{m}\right) d\left(r_{m}, t\right)+a_{m} k_{m} d\left(r_{m}, t\right)+a_{m} k_{m} v_{m} \\
& =\left(1-a_{m}+a_{m} k_{m}\right) d\left(r_{m}, t\right)+a_{m} k_{m} v_{m} \\
& =\left(1-a_{m}\left(1-k_{m}\right)\right) d\left(r_{m}, t\right)+a_{m} k_{m} v_{m} \\
& \leq\left(1-a_{m}\left(1-k_{m}\right)\right)\left(\mathcal{B C} d\left(s_{m}, t\right)+\mathcal{B G}+\mathcal{F}\right)+a_{m} k_{m} v_{m} \\
& d\left(s_{m+1}, t\right) \leq \mathcal{A B C} d\left(s_{m}, t\right)+\mathcal{A B G}+\mathcal{A F}+\mathcal{D}
\end{aligned}
$$

where $\mathcal{A}=1-a_{m}\left(1-k_{m}\right)$ and $\mathcal{D}=a_{m} k_{m} v_{m}$. Since $\sum_{m=1}^{\infty} k_{m}<\infty$ and $\sum_{m=1}^{\infty} v_{m}<$ $\infty$. Therefore $\sum_{m=1}^{\infty} \mathcal{A B C}<\infty$ and $\sum_{m=1}^{\infty} \mathcal{A B G}+\mathcal{A} \mathcal{F}+\mathcal{D}<\infty$. Thus, from Lemma 2.14 and inequality $(8), \lim _{m \rightarrow \infty} d\left(s_{m}, t\right)$ exists and we may assume that

$$
\lim _{m \rightarrow \infty} d\left(s_{m}, t\right)=k \geq 0 .
$$

By (9), $\left\{s_{m}\right\}$ is bounded and therefore $\left\{p_{m}\right\},\left\{q_{m}\right\},\left\{r_{m}\right\},\left\{A^{m} s_{m}\right\},\left\{B^{m} s_{m}\right\}$ and $\left\{C^{m} s_{m}\right\}$ are bounded.

Theorem 3.2. Let $(Y, d)$ be a complete $\mathrm{CAT}(0)$ space and $\mathcal{W}$ be a nonempty closed convex subset of $Y$. Let $\mathcal{T}: \mathcal{W} \rightarrow P(\mathcal{W})$ be multivalued mapping and $P_{\mathcal{T}}$ be a nonexpansive mapping. Let $h: Y \rightarrow(-\infty, \infty]$ be a proper convex and lower semi-continuous function and $A, B, C: \mathcal{W} \rightarrow \mathcal{W}$ be three nearly Lipschitzian mappings with $\left\{k_{m}\right\},\left\{v_{m}\right\}$ being nonnegative real sequences such that $\sum_{m=1}^{\infty} k_{m}<\infty$, 
$\sum_{m=1}^{\infty} v_{m}<\infty$ and $k_{m} \leq 1 \forall m \in N$. Let $\mathcal{E}:=G(A) \cap G(B) \cap G(C) \cap G(\mathcal{T}) \cap$ $\operatorname{argmin}_{r \in \mathcal{W}} h(r) \neq \phi$. Let $\left\{s_{m}\right\}$ be defined by (4). Then

(1) $\lim _{m \rightarrow \infty} d\left(s_{m}, p_{m}\right)=0$;

(2) $\lim _{m \rightarrow \infty} d\left(s_{m}, A s_{m}\right)=\lim _{m \rightarrow \infty} d\left(s_{m}, B s_{m}\right)=\lim _{m \rightarrow \infty} d\left(s_{m}, C s_{m}\right)=0$.

Proof. (1) By Lemma 2.12, we get

$$
\frac{1}{2 \pi_{m}}\left\{d^{2}\left(p_{m}, t\right)-d^{2}\left(s_{m}, t\right)+d^{2}\left(s_{m}, p_{m}\right)\right\} \leq h(t)-h\left(p_{m}\right) .
$$

Since $h(t) \leq h\left(p_{m}\right)$ for each $m \in N$, we have

$$
d^{2}\left(s_{m}, p_{m}\right) \leq d^{2}\left(s_{m}, t\right)-d^{2}\left(p_{m}, t\right) .
$$

From (8) and (9), we have

$$
\begin{aligned}
\liminf _{m \rightarrow \infty} d\left(s_{m+1}, t\right) & \leq \liminf _{m \rightarrow \infty}\left(1-a_{m}\left(1-k_{m}\right)\right) d\left(r_{m}, t\right)+\liminf _{m \rightarrow \infty} a_{m} k_{m} v_{m} \\
k & \leq \liminf _{m \rightarrow \infty} d\left(r_{m}, t\right)
\end{aligned}
$$

From (7), we have

$$
\begin{aligned}
\limsup _{m \rightarrow \infty} d\left(r_{m}, t\right) \leq & \limsup _{m \rightarrow \infty}\left(1-b_{m}\left(1-k_{m}\right)\right)\left(\left(1-c_{m}\left(1-k_{m}\right)\right) d\left(s_{m}, t\right)+c_{m} k_{m} v_{m}\right) \\
& +\limsup _{m \rightarrow \infty}\left(1-b_{m}\right) k_{m} v_{m} \\
\limsup _{m \rightarrow \infty} d\left(r_{m}, t\right) \leq & k .
\end{aligned}
$$

From (11) and (12), we have

$$
\lim _{m \rightarrow \infty} d\left(r_{m}, t\right)=k .
$$

From (7) and (13), we have

$$
\begin{aligned}
\liminf _{m \rightarrow \infty} d\left(r_{m}, t\right) & \leq \liminf _{m \rightarrow \infty}\left(1-b_{m}\left(1-k_{m}\right)\right) d\left(q_{m}, t\right)+\liminf _{m \rightarrow \infty}\left(1-b_{m}\right) k_{m} v_{m} \\
k & \leq \liminf _{m \rightarrow \infty} d\left(q_{m}, t\right)
\end{aligned}
$$

From (6) and (9), we have

$$
\begin{aligned}
\limsup _{m \rightarrow \infty} d\left(q_{m}, t\right) & \leq \limsup _{m \rightarrow \infty}\left(1-c_{m}\left(1-k_{m}\right)\right) d\left(s_{m}, t\right)+\limsup _{m \rightarrow \infty} c_{m} k_{m} v_{m} \\
\limsup _{m \rightarrow \infty} d\left(q_{m}, t\right) & \leq k .
\end{aligned}
$$

From (14) and (15), we have

$$
\lim _{m \rightarrow \infty} d\left(q_{m}, t\right)=k .
$$

From (6) and (16), we have

$$
\begin{aligned}
\liminf _{m \rightarrow \infty} d\left(q_{m}, t\right) & \leq \liminf _{m \rightarrow \infty}\left(1-c_{m}\left(1-k_{m}\right)\right) d\left(p_{m}, t\right)+\liminf _{m \rightarrow \infty} c_{m} k_{m} v_{m} \\
k & \leq \liminf _{m \rightarrow \infty} d\left(p_{m}, t\right) .
\end{aligned}
$$

From (6), we have

$$
\limsup _{m \rightarrow \infty} d\left(p_{m}, t\right) \leq \limsup _{m \rightarrow \infty} d\left(s_{m}, t\right) \leq k .
$$


From (17) and (18), we have

$$
\lim _{m \rightarrow \infty} d\left(p_{m}, t\right)=k
$$

So from (10), we have

$$
\lim _{m \rightarrow \infty} d\left(s_{m}, p_{m}\right)=0
$$

(2) Suppose that $\mathcal{E}$ is nonempty, and let $t \in \mathcal{E}$. From $(9), \lim _{m \rightarrow \infty} d\left(s_{m}, t\right)$ exists and $\left\{s_{m}\right\}$ is bounded. From (4) and Lemma 2.3, we have

$$
\begin{aligned}
d^{2}\left(q_{m}, t\right)= & d^{2}\left(\left(1-c_{m}\right) p_{m} \oplus c_{m} A^{m} z_{m}, t\right) \\
\leq & \left(1-c_{m}\right) d^{2}\left(p_{m}, t\right)+c_{m} d^{2}\left(A^{m} z_{m}, t\right)-c_{m}\left(1-c_{m}\right) d^{2}\left(p_{m}, A^{m} z_{m}\right) \\
\leq & \left(1-c_{m}\right) d^{2}\left(p_{m}, t\right)+c_{m}\left(k_{m} d\left(z_{m}, t\right)+v_{m}\right)^{2}-c_{m}\left(1-c_{m}\right) d^{2}\left(p_{m}, A^{m} z_{m}\right) \\
= & c_{m}\left(k_{m} d\left(z_{m}, t\right)+v_{m}\right)^{2}+\left(1-c_{m}\right) d^{2}\left(p_{m}, t\right)-c_{m}\left(1-c_{m}\right) d^{2}\left(p_{m}, A^{m} z_{m}\right) \\
= & c_{m}\left(k_{m}^{2} d^{2}\left(z_{m}, t\right)+v_{m}^{2}+2 k_{m} v_{m} d\left(z_{m}, t\right)\right)+\left(1-c_{m}\right) d^{2}\left(p_{m}, t\right) \\
& -c_{m}\left(1-c_{m}\right) d^{2}\left(p_{m}, A^{m} z_{m}\right) \\
= & c_{m} k_{m}^{2} d^{2}\left(z_{m}, t\right)+\left(1-c_{m}\right) d^{2}\left(p_{m}, t\right)+c_{m}\left(v_{m}^{2}+2 k_{m} v_{m} d\left(z_{m}, t\right)\right) \\
& -c_{m}\left(1-c_{m}\right) d^{2}\left(p_{m}, A^{m} z_{m}\right) \\
= & c_{m} k_{m}^{2} \operatorname{dist}\left(z_{m}, P_{\mathcal{T}}(t)\right)^{2}+\left(1-c_{m}\right) \operatorname{dist}\left(p_{m}, P_{\mathcal{T}}(t)\right)^{2} \\
& +c_{m}\left(v_{m}^{2}+2 k_{m} v_{m} \operatorname{dist}\left(z_{m}, P_{\mathcal{T}}(t)\right)\right)-c_{m}\left(1-c_{m}\right) d^{2}\left(p_{m}, A^{m} z_{m}\right) \\
= & c_{m} k_{m}^{2} \mathcal{H}\left(P_{\mathcal{T}}\left(p_{m}\right), P_{\mathcal{T}}(t)\right)^{2}+\left(1-c_{m}\right) \mathcal{H}\left(P_{\mathcal{T}}\left(p_{m}\right), P_{\mathcal{T}}(t)\right)^{2} \\
& +c_{m}\left(v_{m}^{2}+2 k_{m} v_{m} \mathcal{H}\left(P_{\mathcal{T}}\left(p_{m}\right), P_{\mathcal{T}}(t)\right)\right)-c_{m}\left(1-c_{m}\right) d^{2}\left(p_{m}, A^{m} z_{m}\right) \\
= & c_{m} k_{m}^{2} d^{2}\left(p_{m}, t\right)+\left(1-c_{m}\right) d^{2}\left(p_{m}, t\right)+c_{m}\left(v_{m}^{2}+2 k_{m} v_{m}\right) d\left(p_{m}, t\right) \\
& -c_{m}\left(1-c_{m}\right) d^{2}\left(p_{m}, A^{m} z_{m}\right) \\
= & c_{m} d^{2}\left(p_{m}, t\right)+\left(1-c_{m}\right) d^{2}\left(p_{m}, t\right)+c_{m}\left(v_{m}^{2}+2 k_{m} v_{m}\right) d\left(p_{m}, t\right) \\
& -c_{m}\left(1-c_{m}\right) d^{2}\left(p_{m}, A^{m} z_{m}\right) \\
= & d^{2}\left(p_{m}, t\right)+c_{m}\left(v_{m}^{2}+2 k_{m} v_{m}\right) d\left(p_{m}, t\right)-c_{m}\left(1-c_{m}\right) d^{2}\left(p_{m}, A^{m} z_{m}\right) \\
= & d^{2}\left(p_{m}, t\right)+p v_{m}-c_{m}\left(1-c_{m}\right) d^{2}\left(p_{m}, A^{m} z_{m}\right) \\
&
\end{aligned}
$$

where $p=c_{m}\left(v_{m}+2 k_{m}\right) d\left(p_{m}, t\right)>0$. Therefore,

$$
c_{m}\left(1-c_{m}\right) d^{2}\left(A^{m} z_{m}, p_{m}\right) \leq d^{2}\left(s_{m}, t\right)-d^{2}\left(q_{m}, t\right)+p v_{m}
$$

Since $\lim _{m \rightarrow \infty} v_{m}=0$, we have $c_{m}\left(1-c_{m}\right) d^{2}\left(A^{m} z_{m}, p_{m}\right)=0$.

From $\liminf _{m \rightarrow \infty} c_{m}\left(1-c_{m}\right)>0$, we have

$$
\lim _{m \rightarrow \infty} d\left(A^{m} z_{m}, p_{m}\right)=0
$$


From (4) and Lemma 2.3, we have

$$
\begin{aligned}
& d^{2}\left(r_{m}, t\right)=d^{2}\left(\left(1-b_{m}\right) B^{m} y_{m} \oplus b_{m} q_{m}, t\right) \\
\leq & \left(1-b_{m}\right) d^{2}\left(B^{m} y_{m}, t\right)+b_{m} d^{2}\left(q_{m}, t\right)-b_{m}\left(1-b_{m}\right) d^{2}\left(B^{m} y_{m}, q_{m}\right) \\
\leq & \left.\left(1-b_{m}\right)\left(k_{m} d\left(y_{m}, t\right)+v_{m}\right)^{2}+b_{m} d^{(} q_{m}, t\right)-b_{m}\left(1-b_{m}\right) d^{2}\left(B^{m} y_{m}, q_{m}\right) \\
= & \left(1-b_{m}\right)\left(k_{m} d\left(y_{m}, t\right)+v_{m}\right)^{2}+b_{m} d^{2}\left(q_{m}, t\right)-b_{m}\left(1-b_{m}\right) d^{2}\left(B^{m} y_{m}, q_{m}\right) \\
= & \left(1-b_{m}\right)\left(k_{m}^{2} d^{2}\left(y_{m}, t\right)+v_{m}^{2}+2 k_{m} v_{m} d\left(y_{m}, t\right)\right)+b_{m} d^{2}\left(q_{m}, t\right) \\
& -b_{m}\left(1-b_{m}\right) d^{2}\left(B^{m} y_{m}, q_{m}\right) \\
= & \left(1-b_{m}\right) k_{m}^{2} d^{2}\left(y_{m}, t\right)+b_{m} d^{2}\left(q_{m}, t\right)+\left(1-b_{m}\right)\left(v_{m}^{2}+2 k_{m} v_{m} d\left(y_{m}, t\right)\right) \\
& -b_{m}\left(1-b_{m}\right) d^{2}\left(B^{m} y_{m}, q_{m}\right) \\
= & \left(1-b_{m}\right) k_{m}^{2} \operatorname{dist}\left(y_{m}, P_{\mathcal{T}}(t)\right)^{2}+b_{m} \operatorname{dist}\left(q_{m}, P_{\mathcal{T}}(t)\right)^{2} \\
& +\left(1-b_{m}\right)\left(v_{m}^{2}+2 k_{m} v_{m} \operatorname{dist}\left(y_{m}, P_{\mathcal{T}}(t)\right)\right)-b_{m}\left(1-b_{m}\right) d^{2}\left(B^{m} y_{m}, q_{m}\right) \\
= & \left(1-b_{m}\right) k_{m}^{2} \mathcal{H}\left(P_{\mathcal{T}}\left(y_{m}\right), P_{\mathcal{T}}(t)\right)^{2}+b_{m} \mathcal{H}\left(P_{\mathcal{T}}\left(q_{m}\right), P_{\mathcal{T}}(t)\right)^{2} \\
& +\left(1-b_{m}\right)\left(v_{m}^{2}+2 k_{m} v_{m} \mathcal{H}\left(P_{\mathcal{T}}\left(q_{m}\right), P_{\mathcal{T}}(t)\right)\right)-b_{m}\left(1-b_{m}\right) d^{2}\left(B^{m} y_{m}, q_{m}\right) \\
= & \left(1-b_{m}\right) k_{m}^{2} d^{2}\left(q_{m}, t\right)+b_{m} d^{2}\left(q_{m}, t\right)+\left(1-b_{m}\right)\left(v_{m}^{2}+2 k_{m} v_{m}\right) d\left(q_{m}, t\right) \\
& -b_{m}\left(1-b_{m}\right) d^{2}\left(B^{m} y_{m}, q_{m}\right) \\
= & \left(1-b_{m}\right) d^{2}\left(q_{m}, t\right)+b_{m} d^{2}\left(q_{m}, t\right)+\left(1-b_{m}\right)\left(v_{m}^{2}+2 k_{m} v_{m}\right) d\left(q_{m}, t\right) \\
& -b_{m}\left(1-b_{m}\right) d^{2}\left(B^{m} y_{m}, q_{m}\right) \\
= & d^{2}\left(q_{m}, t\right)+\left(1-b_{m}\right)\left(v_{m}^{2}+2 k_{m} v_{m}\right) d\left(q_{m}, t\right) \\
& -b_{m}\left(1-b_{m}\right) d^{2}\left(B^{m} y_{m}, q_{m}\right) \\
= & d^{2}\left(q_{m}, t\right)+q v_{m}-b_{m}\left(1-b_{m}\right) d^{2}\left(B^{m} y_{m}, q_{m}\right) \\
&
\end{aligned}
$$

where $q=\left(1-b_{m}\right)\left(v_{m}+2 k_{m}\right) d\left(q_{m}, t\right)>0$. Therefore

$$
b_{m}\left(1-b_{m}\right) d^{2}\left(B^{m} y_{m}, q_{m}\right) \leq d^{2}\left(q_{m}, t\right)-d^{2}\left(r_{m}, t\right)+q v_{m}
$$

Since $\lim _{m \rightarrow \infty} v_{m}=0$, we have

$$
b_{m}\left(1-b_{m}\right) d^{2}\left(B^{m} y_{m}, q_{m}\right)=0 .
$$

From $\liminf \operatorname{in}_{m \rightarrow \infty} b_{m}\left(1-b_{m}\right)>0$, we have

$$
\lim _{m \rightarrow \infty} d\left(B^{m} y_{m}, q_{m}\right)=0
$$


Similarly from (4) and Lemma 2.3, we have

$$
\begin{aligned}
& d^{2}\left(s_{m+1}, t\right)=d^{2}\left(\left(1-a_{m}\right) r_{m} \oplus a_{m} C^{m} x_{m}, t\right) \\
\leq & \left(1-a_{m}\right) d^{2}\left(r_{m}, t\right)+a_{m} d^{2}\left(C^{m} x_{m}, t\right)-a_{m}\left(1-a_{m}\right) d^{2}\left(r_{m}, C^{m} x_{m}\right) \\
\leq & \left(1-a_{m}\right) d^{2}\left(r_{m}, t\right)+a_{m}\left(k_{m} d\left(x_{m}, t\right)+v_{m}\right)^{2}-a_{m}\left(1-a_{m}\right) d^{2}\left(r_{m}, C^{m} x_{m}\right) \\
= & a_{m}\left(k_{m} d\left(x_{m}, t\right)+v_{m}\right)^{2}+\left(1-a_{m}\right) d^{2}\left(r_{m}, t\right)-a_{m}\left(1-a_{m}\right) d^{2}\left(r_{m}, C^{m} x_{m}\right) \\
= & a_{m}\left(k_{m}^{2} d^{2}\left(x_{m}, t\right)+v_{m}^{2}+2 k_{m} v_{m} d\left(x_{m}, t\right)\right)+\left(1-a_{m}\right) d^{2}\left(r_{m}, t\right) \\
& -a_{m}\left(1-a_{m}\right) d^{2}\left(r_{m}, C^{m} x_{m}\right) \\
= & a_{m} k_{m}^{2} d^{2}\left(x_{m}, t\right)+\left(1-a_{m}\right) d^{2}\left(r_{m}, t\right)+a_{m}\left(v_{m}^{2}+2 k_{m} v_{m} d\left(x_{m}, t\right)\right) \\
& -a_{m}\left(1-a_{m}\right) d^{2}\left(r_{m}, C^{m} x_{m}\right) \\
= & a_{m} k_{m}^{2} \operatorname{dist}\left(x_{m}, P_{\mathcal{T}}(t)\right)^{2}+\left(1-a_{m}\right) \operatorname{dist}\left(r_{m}, P_{\mathcal{T}}(t)\right)^{2}+a_{m}\left(v_{m}^{2}\right. \\
& \left.+2 k_{m} v_{m} \operatorname{dist}\left(x_{m}, P_{\mathcal{T}}(t)\right)\right)-a_{m}\left(1-a_{m}\right) d^{2}\left(r_{m}, C^{m} x_{m}\right) \\
= & a_{m} k_{m}^{2} \mathcal{H}\left(P_{\mathcal{T}}\left(r_{m}\right), P_{\mathcal{T}}(t)\right)^{2}+\left(1-a_{m}\right) \mathcal{H}\left(P_{\mathcal{T}}\left(r_{m}\right), P_{\mathcal{T}}(t)\right)^{2}+a_{m}\left(v_{m}^{2}\right. \\
& \left.+2 k_{m} v_{m} \mathcal{H}\left(P_{\mathcal{T}}\left(r_{m}\right), P_{\mathcal{T}}(t)\right)\right)-a_{m}\left(1-a_{m}\right) d^{2}\left(r_{m}, C^{m} x_{m}\right) \\
= & a_{m} k_{m}^{2} d^{2}\left(r_{m}, t\right)+\left(1-a_{m}\right) d^{2}\left(r_{m}, t\right)+a_{m}\left(v_{m}^{2}+2 k_{m} v_{m}\right) d\left(r_{m}, t\right) \\
& -a_{m}\left(1-a_{m}\right) d^{2}\left(r_{m}, C^{m} x_{m}\right) \\
= & a_{m} d^{2}\left(r_{m}, t\right)+\left(1-a_{m}\right) d^{2}\left(r_{m}, t\right)+a_{m}\left(v_{m}^{2}+2 k_{m} v_{m}\right) d\left(r_{m}, t\right) \\
& -a_{m}\left(1-a_{m}\right) d^{2}\left(r_{m}, C^{m} x_{m}\right) \\
= & d^{2}\left(r_{m}, t\right)+a_{m}\left(v_{m}^{2}+2 k_{m} v_{m}\right) d\left(r_{m}, t\right) \\
& -a_{m}\left(1-a_{m}\right) d^{2}\left(r_{m}, C^{m} x_{m}\right) \\
= & d^{2}\left(r_{m}, t\right)+r v_{m}-a_{m}\left(1-a_{m}\right) d^{2}\left(r_{m}, C^{m} x_{m}\right) \\
&
\end{aligned}
$$

where $r=a_{m}\left(v_{m}+2 k_{m}\right) d\left(r_{m}, t\right)>0$. Therefore

$$
a_{m}\left(1-a_{m}\right) d^{2}\left(C^{m} x_{m}, r_{m}\right) \leq d^{2}\left(r_{m}, t\right)-d^{2}\left(s_{m+1}, t\right)+r v_{m} .
$$

Since $\lim _{m \rightarrow \infty} v_{m}=0$, we have

$$
a_{m}\left(1-a_{m}\right) d^{2}\left(C^{m} x_{m}, r_{m}\right)=0 .
$$

From $\liminf \operatorname{in}_{m \rightarrow \infty} a_{m}\left(1-a_{m}\right)>0$, we have

$$
\lim _{m \rightarrow \infty} d\left(C^{m} x_{m}, r_{m}\right)=0 .
$$

From (20) and (21), we have

$$
\begin{aligned}
d\left(q_{m}, s_{m}\right) & =d\left(\left(1-c_{m}\right) p_{m} \oplus c_{m} A^{m} z_{m}, s_{m}\right) \\
& \leq\left(1-c_{m}\right) d\left(p_{m}, s_{m}\right) \oplus c_{m} d\left(A^{m} z_{m}, s_{m}\right) \\
& \leq\left(1-c_{m}\right) d\left(p_{m}, s_{m}\right) \oplus c_{m}\left(d\left(A^{m} z_{m}, p_{m}\right)+d\left(p_{m}, s_{m}\right)\right) \\
& \left.=\left(1-c_{m}\right) d\left(p_{m}, s_{m}\right) \oplus c_{m} d\left(A^{m} z_{m}, p_{m}\right)+c_{m} d\left(p_{m}, s_{m}\right)\right) \\
& =d\left(p_{m}, s_{m}\right) \oplus c_{m} d\left(A^{m} z_{m}, p_{m}\right) \\
& \rightarrow 0 \text { as } m \rightarrow \infty .
\end{aligned}
$$


From (22) and (24), we have

$$
\begin{aligned}
d\left(r_{m}, s_{m}\right) & =d\left(\left(1-b_{m}\right) q_{m} \oplus b_{m} B^{m} y_{m}, s_{m}\right) \\
\leq & \left(1-b_{m}\right) d\left(q_{m}, s_{m}\right) \oplus b_{m} d\left(B^{m} y_{m}, s_{m}\right) \\
\leq & \left(1-b_{m}\right) d\left(q_{m}, s_{m}\right) \oplus b_{m}\left(d\left(B^{m} y_{m}, q_{m}\right)+d\left(q_{m}, s_{m}\right)\right) \\
& \left.=\left(1-b_{m}\right) d\left(q_{m}, s_{m}\right) \oplus b_{m} d\left(B^{m} y_{m}, q_{m}\right)+b_{m} d\left(q_{m}, s_{m}\right)\right) \\
& =d\left(q_{m}, s_{m}\right) \oplus b_{m} d\left(B^{m} y_{m}, q_{m}\right) \\
& \rightarrow 0 \text { as } m \rightarrow \infty .
\end{aligned}
$$

From (23) and (25), we have

$$
\begin{aligned}
d\left(s_{m+1}, s_{m}\right) & =d\left(\left(1-a_{m}\right) r_{m} \oplus a_{m} C^{m} x_{m}, s_{m}\right) \\
& \leq\left(1-a_{m}\right) d\left(r_{m}, s_{m}\right) \oplus a_{m} d\left(C^{m} x_{m}, s_{m}\right) \\
& \leq\left(1-a_{m}\right) d\left(r_{m}, s_{m}\right) \oplus a_{m}\left(d\left(C^{m} x_{m}, r_{m}\right)+d\left(r_{m}, s_{m}\right)\right) \\
& \left.=\left(1-a_{m}\right) d\left(r_{m}, s_{m}\right) \oplus a_{m} d\left(C^{m} x_{m}, r_{m}\right)+a_{m} d\left(r_{m}, s_{m}\right)\right) \\
& =d\left(r_{m}, s_{m}\right) \oplus a_{m} d\left(C^{m} x_{m}, r_{m}\right) \\
& \rightarrow 0 \text { as } m \rightarrow \infty .
\end{aligned}
$$

By the triangular inequality, (20) and (21),

$$
\begin{aligned}
d\left(A^{m} s_{m}, s_{m}\right) & \leq d\left(A^{m} s_{m}, A^{m} z_{m}\right)+d\left(A^{m} z_{m}, p_{m}\right)+d\left(p_{m}, s_{m}\right) \\
& \leq k_{m}\left(d\left(s_{m}, z_{m}\right)+v_{m}\right)+d\left(A^{m} z_{m}, p_{m}\right)+d\left(p_{m}, s_{m}\right) \\
& \leq k_{m}\left(\mathcal{H}\left(P_{\mathcal{T}} s_{m}, P_{\mathcal{T}} z_{m}\right)+v_{m}\right)+d\left(A^{m} z_{m}, p_{m}\right)+d\left(p_{m}, s_{m}\right) \\
& \leq k_{m}\left(d\left(s_{m}, p_{m}\right)+v_{m}\right)+d\left(A^{m} z_{m}, p_{m}\right)+d\left(p_{m}, s_{m}\right) \\
& \rightarrow 0 \text { as } m \rightarrow \infty .
\end{aligned}
$$

By the triangular inequality, (22) and (24),

$$
\begin{aligned}
d\left(B^{m} s_{m}, s_{m}\right) & \leq d\left(B^{m} s_{m}, B^{m} y_{m}\right)+d\left(B^{m} y_{m}, q_{m}\right)+d\left(q_{m}, s_{m}\right) \\
& \leq k_{m}\left(d\left(s_{m}, y_{m}\right)+v_{m}\right)+d\left(B^{m} y_{m}, q_{m}\right)+d\left(q_{m}, s_{m}\right) \\
& \leq k_{m}\left(\mathcal{H}\left(P_{\mathcal{T}} s_{m}, P_{\mathcal{T}} y_{m}\right)+v_{m}\right)+d\left(B^{m} y_{m}, q_{m}\right)+d\left(q_{m}, s_{m}\right) \\
& \leq k_{m}\left(d\left(s_{m}, q_{m}\right)+v_{m}\right)+d\left(B^{m} y_{m}, q_{m}\right)+d\left(q_{m}, s_{m}\right) \\
& \rightarrow 0 \text { as } m \rightarrow \infty .
\end{aligned}
$$

By the triangular inequality, (23) and (25),

$$
\begin{aligned}
d\left(C^{m} s_{m}, s_{m}\right) & \leq d\left(C^{m} s_{m}, C^{m} x_{m}\right)+d\left(C^{m} x_{m}, r_{m}\right)+d\left(r_{m}, s_{m}\right) \\
& \leq k_{m}\left(d\left(s_{m}, x_{m}\right)+v_{m}\right)+d\left(C^{m} y_{m}, r_{m}\right)+d\left(r_{m}, s_{m}\right) \\
& \leq k_{m}\left(\mathcal{H}\left(P_{\mathcal{T}} s_{m}, P_{\mathcal{T}} x_{m}\right)+v_{m}\right)+d\left(C^{m} x_{m}, r_{m}\right)+d\left(r_{m}, s_{m}\right) \\
& \leq k_{m}\left(d\left(s_{m}, r_{m}\right)+v_{m}\right)+d\left(C^{m} x_{m}, r_{m}\right)+d\left(r_{m}, s_{m}\right) \\
& \rightarrow 0 \text { as } m \rightarrow \infty .
\end{aligned}
$$


From (26) and (27), we have

$$
\begin{aligned}
d\left(s_{m}, A s_{m}\right) \leq & d\left(s_{m}, s_{m+1}\right)+d\left(s_{m+1}, A^{m+1} s_{m+1}\right)+d\left(A^{m+1} s_{m+1}, A^{m+1} s_{m}\right) \\
& +d\left(A^{m+1} s_{m}, A s_{m}\right) \\
\leq & d\left(s_{m}, s_{m+1}\right)+d\left(s_{m+1}, A^{m+1} s_{m+1}\right)+k_{m}\left(d\left(s_{m+1}, s_{m}\right)+v_{m}\right) \\
& +k_{m}\left(d\left(A^{m} s_{m}, s_{m}\right)+v_{m}\right) \\
\rightarrow & 0 \text { as } m \rightarrow \infty .
\end{aligned}
$$

From (26) and (28), we have

$$
\begin{aligned}
d\left(s_{m}, B s_{m}\right) \leq & d\left(s_{m}, s_{m+1}\right)+d\left(s_{m+1}, B^{m+1} s_{m+1}\right)+d\left(B^{m+1} s_{m+1}, B^{m+1} s_{m}\right) \\
& +d\left(B^{m+1} s_{m}, B s_{m}\right) \\
\leq & d\left(s_{m}, s_{m+1}\right)+d\left(s_{m+1}, B^{m+1} s_{m+1}\right)+k_{m}\left(d\left(s_{m+1}, s_{m}\right)+v_{m}\right) \\
& +k_{m}\left(d\left(B^{m} s_{m}, s_{m}\right)+v_{m}\right) \\
\rightarrow & 0 \text { as } m \rightarrow \infty .
\end{aligned}
$$

From (26) and (29), we have

$$
\begin{aligned}
d\left(s_{m}, C s_{m}\right) \leq & d\left(s_{m}, s_{m+1}\right)+d\left(s_{m+1}, C^{m+1} s_{m+1}\right)+d\left(C^{m+1} s_{m+1}, C^{m+1} s_{m}\right) \\
& +d\left(C^{m+1} s_{m}, C s_{m}\right) \\
\leq & d\left(s_{m}, s_{m+1}\right)+d\left(s_{m+1}, C^{m+1} s_{m+1}\right)+k_{m}\left(d\left(s_{m+1}, s_{m}\right)+v_{m}\right) \\
& +k_{m}\left(d\left(B^{m} s_{m}, s_{m}\right)+v_{m}\right) \\
\rightarrow & 0 \text { as } m \rightarrow \infty .
\end{aligned}
$$

Theorem 3.3. Let $(Y, d)$ be a complete $\mathrm{CAT}(0)$ space and $\mathcal{W}$ be a nonempty closed convex subset of $Y$. Let $\mathcal{T}: \mathcal{W} \rightarrow P(\mathcal{W})$ be multivalued mapping and $P_{\mathcal{T}}$ be a nonexpansive mapping. Let $h: Y \rightarrow(-\infty, \infty]$ be a proper convex and lower semicontinuous function, $A, B, C: \mathcal{W} \rightarrow \mathcal{W}$ be three nearly Lipschitzian mappings. Then $\left\{s_{m}\right\}$ defined in (4) is $\Delta$-convergent to a common fixed point of $\mathcal{E}$.

Proof. From Lemma 2.13 and (20), we have

$$
\begin{aligned}
d\left(\mathcal{J} s_{m}, s_{m}\right) & \leq d\left(\mathcal{J} s_{m}, p_{m}\right)+d\left(p_{m}, s_{m}\right) \\
& =d\left(\mathcal{J} s_{m}, \mathcal{J}_{\pi_{m}} p_{m}\right)+d\left(p_{m}, s_{m}\right) \\
& =d\left(\mathcal{J} s_{m}, \mathcal{J}_{\pi}\left(\frac{\pi_{m}-\pi}{\pi_{m}} \mathcal{J}_{\pi_{m}} s_{m} \oplus \frac{\pi}{\pi_{m}}\right)\right)+d\left(p_{m}, s_{m}\right) \\
& \leq d\left(s_{m},\left(1-\frac{\pi}{\pi_{m}}\right) \mathcal{J}_{\pi_{m}} s_{m} \oplus \frac{\pi}{\pi_{m}} s_{m}\right)+d\left(p_{m}, s_{m}\right) \\
& \leq\left(1-\frac{\pi}{\pi_{m}}\right) d\left(s_{m}, \mathcal{J}_{\pi_{m}} s_{m}\right)+\frac{\pi}{\pi_{m}} d\left(s_{m}, s_{m}\right)+d\left(p_{m}, s_{m}\right) \\
& \leq\left(1-\frac{\pi}{\pi_{m}}\right) d\left(s_{m}, p_{m}\right)+d\left(p_{m}, s_{m}\right) \\
& \rightarrow 0 \quad \text { as } m \rightarrow \infty
\end{aligned}
$$


By Theorem 3.1, we have $\lim _{m \rightarrow \infty} d\left(s_{m}, t\right)$ exists for all $t \in \mathcal{E}$ and

$$
\lim _{m \rightarrow \infty} d\left(s_{m}, A s_{m}\right)=\lim _{m \rightarrow \infty} d\left(s_{m}, B s_{m}\right)=\lim _{m \rightarrow \infty} d\left(s_{m}, C s_{m}\right)=0 .
$$

Now we have to show that

$$
\mathcal{W}_{\triangle}\left(s_{m}\right)=\cup_{\left\{u_{m}\right\} \subset\left\{s_{m}\right\}} \mathcal{A}\left(\left\{u_{m}\right\}\right) \subset \mathcal{E} .
$$

Let $u \in \mathcal{W}_{\triangle}\left(s_{m}\right)$. Then there exists a subsequence $\left\{u_{m}\right\}$ of $\left\{s_{m}\right\}$ such that $\left.\mathcal{A}\left(u_{m}\right\}\right)=$ $\{u\}$. From Definition 2.2, there exists a subsequence $\left\{v_{m}\right\}$ of $\left\{u_{m}\right\}$ such that $\Delta-\lim _{m \rightarrow \infty} v_{m}=v$ for some $v \in \mathcal{W}$. By Lemma 2.5, $v \in \mathcal{E}$. By Lemma 2.4, $u=v$. This shows that $\mathcal{W}_{\triangle}\left(\left\{s_{m}\right\}\right) \subset \mathcal{E}$.

Now we have to prove that the sequence $\left\{s_{m}\right\} \Delta$-converges to a point in $\mathcal{E}$, which will prove that $\mathcal{W}_{\triangle}\left(\left\{s_{m}\right\}\right)$ consists of exactly one point. Let $\left\{u_{m}\right\}$ be a subsequence of $\left\{s_{m}\right\}$ with $\left.\mathcal{A}\left(u_{m}\right\}\right)=\{u\}$, and let $\left.\mathcal{A}\left(s_{m}\right\}\right)=\{s\}$. Since $u \in \mathcal{W}_{\triangle}\left(\left\{s_{m}\right\}\right) \subset \mathcal{E}$ and $\left\{d\left(s_{m}, u\right)\right\}$ converges by Lemma 2.4, we have $s=u$. Therefore $\mathcal{W}_{\triangle}\left(\left\{s_{m}\right\}\right)=$ $\{s\}$.

Corollary 3.4. Let $(Y, d)$ be a complete $\mathrm{CAT}(0)$ space and $\mathcal{W}$ be a nonempty closed convex subset of $Y$. Let $\mathcal{T}: \mathcal{W} \rightarrow P(\mathcal{W})$ be multivalued mapping and $P_{\mathcal{W}}$ be a nonexpansive mapping. Let $h: Y \rightarrow(-\infty, \infty]$ be a proper convex and lower semicontinuous function and $A, B, C: \mathcal{W} \rightarrow \mathcal{W}$ be three nearly Lipschitzian mappings and $z_{m} \in P_{\mathcal{T}}\left(p_{m}\right), y_{m} \in P_{\mathcal{T}}\left(q_{m}\right), x_{m} \in P_{\mathcal{T}}\left(r_{m}\right),\left\{a_{m}\right\},\left\{b_{m}\right\},\left\{c_{m}\right\}$ and $\pi_{m}$ satisfy all the conditions of Theorem 3.1. Let $\left\{s_{m}\right\}$ be sequence defined by (4). Then the sequence $\left\{s_{m}\right\}$ converges weakly to a common point in $\mathcal{E}$.

Now we construct and prove strong convergence theorems.

Let $\mathcal{W}$ be a nonempty closed convex subset of $\operatorname{CAT}(0)$ space $(Y, d)$. A family $\{A, B, C, \mathcal{T}\}$ of mappings is said to satisfy Condition $(\mathcal{E})$, if there exists a nondecreasing function $h:[0, \infty) \rightarrow[0, \infty)$ with $h(0)=0$ and $h(w)>0$ for all $w \in(0, \infty)$ such that

$$
d(s, A s) \geq h(d(s, \mathcal{G}))
$$

or

$$
d(s, B s) \geq h(d(s, \mathcal{G}))
$$

or

$$
d(s, C s) \geq h(d(s, \mathcal{G}))
$$

or

$$
d(s, T s) \geq h(d(s, \mathcal{G})),
$$

for all $s \in Y$, where $\mathcal{G}=G(A) \cap G(B) \cap G(C) \cap G(\mathcal{T})$.

Theorem 3.5. Let $(Y, d)$ be a complete $\mathrm{CAT}(0)$ space and $\mathcal{W}$ be a nonempty closed convex subset of $Y$. Let $\mathcal{T}: \mathcal{W} \rightarrow P(\mathcal{W})$ be multivalued mapping and $P_{\mathcal{T}}$ be a nonexpansive mapping. Let $h: Y \rightarrow(-\infty, \infty]$ be a proper convex and lower semicontinuous function and $A, B, C: \mathcal{W} \rightarrow \mathcal{W}$ be three nearly Lipschitzian mappings and $z_{m} \in P_{\mathcal{T}}\left(p_{m}\right), y_{m} \in P_{\mathcal{T}}\left(q_{m}\right), x_{m} \in P_{\mathcal{T}}\left(r_{m}\right),\left\{a_{m}\right\},\left\{b_{m}\right\},\left\{c_{m}\right\}$ and $\left\{\pi_{m}\right\}$ satisfy all the conditions of Theorem 3.1 and $\left\{A, B, C, \mathcal{J}_{\pi}\right\}$ satisfy the Condition $(\mathcal{E})$. Then the sequence $\left\{s_{m}\right\}$ defined in (4) strongly converges to an element of $\mathcal{E}$. 
Proof. From Theorem 3.1, we have $\lim _{m \rightarrow \infty} d\left(s_{m}, t\right)$ exists for all $t \in \mathcal{E}$. Also it follows that $\lim _{m \rightarrow \infty} d\left(s_{m}, \mathcal{E}\right)$ exists. On the otherhand, by Condition $(\mathcal{E})$, we have

$$
\lim _{m \rightarrow \infty} d\left(d\left(s_{m}, \mathcal{E}\right)\right) \geq \lim _{m \rightarrow \infty} d\left(s_{m}, A s_{m}\right)=0 .
$$

or

$$
\lim _{m \rightarrow \infty} d\left(d\left(s_{m}, \mathcal{E}\right)\right) \geq \lim _{m \rightarrow \infty} d\left(s_{m}, B s_{m}\right)=0 .
$$

or

$$
\lim _{m \rightarrow \infty} d\left(d\left(s_{m}, \mathcal{E}\right)\right) \geq \lim _{m \rightarrow \infty} d\left(s_{m}, C s_{m}\right)=0 .
$$

or

$$
\lim _{m \rightarrow \infty} d\left(d\left(s_{m}, \mathcal{E}\right)\right) \geq \lim _{m \rightarrow \infty} d\left(s_{m}, \mathcal{J}_{\pi} s_{m}\right)=0 .
$$

Thus, we have $\lim _{m \rightarrow \infty} d\left(s_{m}, \mathcal{E}\right)=0$. Using the property of $h$, we have

$$
\lim _{m \rightarrow \infty} d\left(s_{m}, \mathcal{E}\right)=0 .
$$

Therefore, $\left\{s_{m}\right\}$ is a Cauchy sequence in $Y$, and so $\left\{s_{m}\right\}$ converges to a point $t \in Y$ and hence $d(t, \mathcal{E})=0$. Since $\mathcal{E}$ is closed, so we have $t \in \mathcal{E}$.

Remark 3.6. (1) Our results extends the results of Hussain et al. [22] in the framework of CAT(0) spaces. They established convergence theorems for different classes of generalized nonexpansive mappings including a total asymptotically nonexpansive mapping, a multivalued mapping, and a minimizer of a convex function for solving the convex minimization problem and the common fixed point problem.

(2) Our results is generalization of the results of Pakkaranang et al. [33] in the framework of $\mathrm{CAT}(0)$ spaces. They established convergence theorems for three asymptotically quasinonexpansive mappings involving the convex and lower semi-continuous function for solving the convex minimization problem and the common fixed point problem.

\section{Numerical Examples}

In this section, we discuss a numerical result to illustrate the convergence of the iterative algorithm (4) to support our example.

Example 4.1. Consider $Y=R$ with its usual metric, then $Y$ is complete $\mathrm{CAT}(0)$ space (see [35, Example 3]). Assume that $C=[0,5000]$. Here $C$ is closed and bounded subset of $Y$. Let $\mathcal{T}: C \rightarrow P(C)$ be a mapping defined by

$$
\mathcal{T}(s)=\left\{\frac{3 s+4}{5}\right\} \quad \forall s \in C .
$$

It is clear that the mapping $\mathcal{T}$ is nonexpansive. 
TABLE 1. Numerical values of $s_{m},\left\|s_{m}-s_{m-1}\right\|_{2}$ and $h\left(s_{m}\right)$

\begin{tabular}{llll}
\hline No. of iterations & $s_{m}$ & $\left\|s_{m}-s_{m-1}\right\|_{2}$ & $h\left(s_{m}\right)$ \\
\hline $\mathrm{m}=1$ & 50 & - & 1269.2 \\
$\mathrm{~m}=2$ & 2.59511 & 47.4049 & 3.60534 \\
$\mathrm{~m}=3$ & 1.99745 & 0.59766 & 1.99389 \\
$\mathrm{~m}=4$ & 1.99841 & 0.00095 & 1.99618 \\
$\mathrm{~m}=5$ & 1.99903 & 0.00062 & 1.99767 \\
$\mathrm{~m}=6$ & 1.99931 & 0.00028 & 1.99835 \\
$\mathrm{~m}=7$ & 1.99947 & 0.00015 & 1.99872 \\
$\mathrm{~m}=8$ & 1.99956 & 0.00009 & 1.99895 \\
$\mathrm{~m}=9$ & 1.99962 & 0.00006 & 1.99910 \\
$\mathrm{~m}=10$ & 1.99967 & 0.00004 & 1.99921 \\
$\mathrm{~m}=11$ & 1.99970 & 0.00003 & 1.99929 \\
$\mathrm{~m}=12$ & 1.99973 & 0.00003 & 1.99935 \\
$\mathrm{~m}=13$ & 1.99975 & 0.00002 & 1.99940 \\
$\mathrm{~m}=14$ & 1.99977 & 0.00002 & 1.99944 \\
$\mathrm{~m}=15$ & 1.99978 & 0.00001 & 1.99947 \\
$\mathrm{~m}=16$ & 1.99979 & 0.00001 & 1.99950 \\
$\mathrm{~m}=17$ & 1.99980 & 0.00000 & 1.99952 \\
$\mathrm{~m}=18$ & 1.99981 & 0.00000 & 1.99954 \\
$\mathrm{~m}=19$ & 1.99981 & 0.00000 & 1.99956 \\
$\mathrm{~m}=20$ & 1.99981 & 0.00000 & 1.99956 \\
\hline
\end{tabular}

Now we define a mapping $h: Y \rightarrow(-\infty, \infty]$ such that

$$
h(s)=\|s\|_{1}+\frac{1}{2}\|s\|_{2}^{2}-\frac{3}{5} s-\frac{4}{5}
$$

It is easy to check that $h$ is a proper convex and lower semi-continuous function and consider the nearly Lipschitzian mappings $A, B, C$ from definitions (2.8), (2.9) and (2.10), respectively and $\mathcal{T}$ is nonexpansive mapping with $G(A) \cap G(B) \cap$ $G(C) \cap G(\mathcal{T})=\{2\}$. Suppose that $a_{m}=\frac{15 m-3}{16 m}, b_{m}=\frac{m+5}{22 m}$ and $c_{m}=\frac{33 m-7}{34 m}$ and $s_{1}=50$ is the initial value. We obtain the numerical results with the errors values in Table 1. From Table 1, Figure 1 and Figure 2, it is clear that the sequence $\left\{s_{m}\right\}$ converges to $1.99999 \equiv 2$ which is common fixed point of solution of a minimizer of a function $h$, multivalued mapping $\mathcal{T}$ and three nearly Lipschtzian mappings $A, B$ and $C$.

\section{Conclusion}

In this paper, we proved the $\Delta$-convergence, strong and weak convergence results for the modified proximal point algorithm for three nearly Lipschitzian asymptotically nonexpansive mappings and multivalued mapping in CAT(0) space. Also, we illustrated the efficiency of modified proximal point algorithm by numerical 


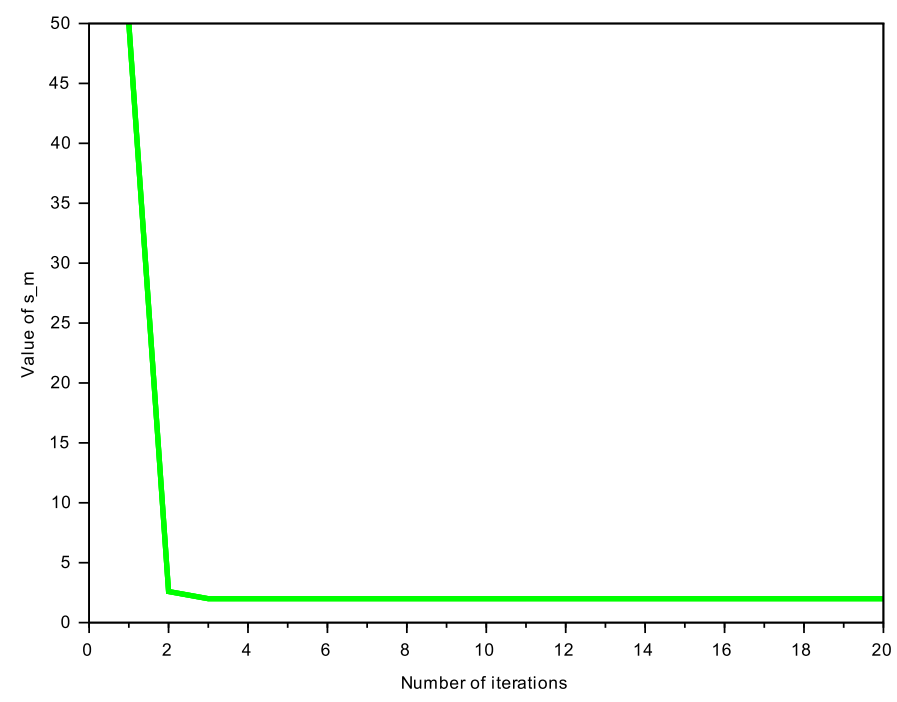

Figure 1. Value of $s_{m}$

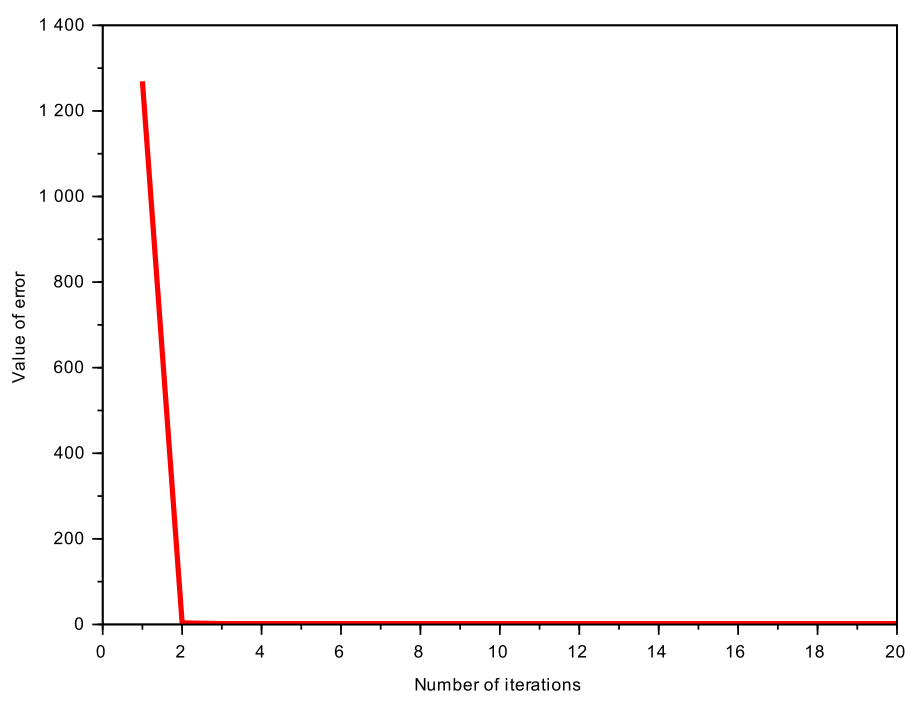

FIgURE 2. Value of error 
example in $\mathrm{CAT}(0)$ space for supporting our results. The results in this paper generalized the results of Hussain et al. [22] and Pakkaranang et al. [33].

\section{REFERENCES}

[1] Abbas, M., Ibrahim, Y., Khan, A.R. and De la Sen, M., Split Variational Inclusion Problem and Fixed Point Problem for a Class of Multivalued Mappings in CAT(0) Spaces," Mathematics, 2019 7: 749.

[2] Abbas, M., Sahu, D.R. and Kadelburg, Z., Fixed point theorems for Lipschitzian type mappings in CAT(0) spaces", Mathematical and Computer Modelling, 55 (2012), 1418-1427.

[3] Ambrosio, L., Gigli, N. and Savare, G. Gradient Flows in Metric Spaces and in the Space of Probability Measures, 2nd edn. Lectures in Mathematics ETH Zrich, Birkhuser, Basel, 2008.

[4] Ansari, Q.H., Babu, F. and Yao, J., Regularization of proximal point algorithm in Hadamard manifolds", Journal of Fixed Point Theory, (2019), 21:25.

[5] Ansari, Q.H. and Babu, F., Proximal point algorithm for inclusion problems in Hadamard manifolds with applications", Optim. Lett., (2019), 1:21.

[6] Ahmadi, P. and Khatibzadeh, H., On the convergence of inexact proximal point algorithm on Hadamard manifolds", Taiwanese J. Math., 18 (2014), 419-433.

[7] Ariza-Ruiz, D., Leustean, L. and Lopez, G., Firmly nonexpansive mappings in classes of geodesic spaces", Trans. Am. Math. Soc., 366 (2014), 4299-4322.

[8] Bacak, M., The proximal point algorithm in metric spaces", Isr. J. Math., 194 (2013), 689701.

[9] Bacak, M. and Reich, S., The asymptotic behavior of a class of nonlinear semigroups in Hadamard spaces", J. Fixed Point Theory Appl., 16 (2014), 189202.

[10] Bento, G.C., CruzNeto, J.X. and Oliveira, P.R., A new approach to the proximal point method: Convergence on general riemannian manifolds", J. Optim. Theory Appl., 168 (2016), 743755 .

[11] Bento, G.C. and Ferreira, O.P. and Pereira, Y.R.L, Proximal point method for vector optimization on Hadamard manifold", Operation Research Letters, 46(1) (2018), 13:18.

[12] Boikanyo, O.A. and Morosanu, G., A proximal point algorithm converging strongly for general errors", Optim. Lett., 4 (2010) 635-641.

[13] Bruck, R.E. and Reich, S., Nonexpansive projections and resolvents of accretive operators in Banach spaces", Houston J. Math., 3 (1977), 459-470.

[14] Chang, S.S., Yao, J.C., Wang, L. and Qin, L.J., Some convergence theorems involving proximal point and common fixed points for asymptotically nonexpansive mappings in $\operatorname{CAT}(0)$ spaces", Fixed Point Theory Appl., 68 (2016) : 2016.

[15] Cholamjiak, P. and Abdou, A.A. and Cho, Y.J., Proximal point algorithms involving fixed points of nonexpansive mappings in CAT(0) spaces", Fixed Point Theory Appl., 227 (2015), 113.

[16] Cholamjiak, P., The modified proximal point algorithm in CAT(0) Space", Optim. Lett., 9 (2015), 1401-1410.

[17] Cholamjiak, P., Cho, Y.J. and Suantai, S., Composite iterative schemes for maximal monotone operators in reflexive Banach spaces," Fixed Point Theory Appl. (2011) 2011:7.

[18] Cholamjiak, W., Cholamjiak, P. and Suantai, S., An inertial forwardbackward splitting method for solving inclusion problems in Hilbert spaces", J. Fixed Point Theory Appl., (2018) 20: 42.

[19] De la Sen, M., About fixed points in CAT(0) spaces under a combined structure of two self-mappings, Journal of Mathematics, 2017, Article ID 1470582, 13 pages, 2017.

[20] Ferreira, O.P., Oliveira, P.R., Proximal point algorithm on Riemannian manifolds", Optimization, 51 (2002), 257-270. 
[21] Guler, O., On the convergence of the proximal point algorithm for convex minimization", SIAM J. Control Optim. 29 (1991), 403-419.

[22] Hussain, S. and Singh, N., $\Delta-$ convergence for proximal point algorithm and fixed point problem in CAT(0) space", Fixed Point Theory Appl., 8 (2019) : 2019.

[23] Jost, J., Convex functionals and generalized harmonic maps into spaces of nonpositive curvature", Comment. Math. Helv., 70 (1995), 659673.

[24] Kamimura, S. and Takahashi, W., Approximating solutions of maximal monotone operators in Hilbert spaces", J. Approx. Theory, 106 (2000), 226-240.

[25] Kesornprom, S. and Cholamjiak, P., Proximal type algorithms involving linesearch and inertial techniques for split variational inclusion problem in Hilbert spaces with applications," Optimization (2019) DOI : 10.1080/02331934.2019.1638389

[26] Kim, J.K., Dashputre, S. and Das, A.K., Convergence theorems of S-iteration process for Lipschitzian type multi-valued mappings in Banach Spaces", Global Journal of Pure and Applied Mathematics, 13(1) (2017), 121-135.

[27] Li, C., Lopez, G.and Martin-Marquez, V., Monotone vector fields and the proximal point algorithm on Hadamard manifolds", J. London Math. Soc., 79 (2009), 663683.

[28] Markin, J.T., Continuous dependence of fixed point sets", Proc. Am. Math. Soc., 38 (1973), 545-547.

[29] Martinet, B., Regularisation dinquations variationnelles par approximations successives", Rev. Fr. Inf. Rech. Oper., 4 (1970), 154-158.

[30] Mayer, U.F., Gradient flows on nonpositively curved metric spaces and harmonic maps", Commun. Anal. Geom., 6 (1998), 199253.

[31] Nadler, S.B., Multivalued contraction mappings", Pac. J. Math. 30 (1969), 475-488.

[32] Nanjaras B. and Panyanak B., Demiclosed principle for asymptotically nonexpansive mappings in CAT(0) spaces, Fixed Point theory and Applications, 2010, article number 268780, 2010.

[33] Pakkaranang, N., Kumam, P. and Cho, Y.J., Proximal point algorithms for solving convex minimization problem and common fixed points problem of asymptotically quasinonexpansive mappings in CAT(0) spaces with convergence analysis", Numer. Algorithms, 78(3) (2018), 827-845.

[34] Phuengrattana, W., Onjai-uea, N. and Cholamjiak, P., Modified proximal point algorithms for solving constrained minimization and fixed point problems in complete CAT(0) spaces", Mediterr. J. Math. 2018 (2018), 15:97.

[35] Rastgoo, M. and Abkar, A., A new iteration process for approximation of fixed points of mean nonexpansive mappings in CAT(0) spaces", Cogent Mathematics 2017 4: 1396642.

[36] Reich, S. and Saback, S., Two strong convergence theorems for a proximal method in reflexive Banach spaces", Numer. Funct. Anal. Optim., 31 (2010), 2244.

[37] Reich, S. and Salinas, Z., Weak convergence of infinite products of operators in Hadamard spaces", Rend. Circ. Mat. Palermo, 65 (2016), 5571.

[38] Rockafeller, R.T., Monotone operators and the proximal point algorithm", SIAM J. Control Optim., 14 (1976), 877-898.

[39] Shimizu, T. and Takahashi, W., Fixed points of multivalued mappings in certain convex metric space", Topol. Methods Nonlinear Analysis, 8 (1996), 197-203.

[40] Suparatulatorn, R., Cholamjiak, P. and Suantai, S., On solving the minimization problem and the fixed-point problem for nonexpansive mappings in CAT(0) spaces", Optim. Methods Softw., 32 (2017), 182192.

[41] Wang, J., Li, C., Lopez, G. and Yao, J., Convergence analysis of inexact proximal point algorithms on Hadamard manifolds", J. Glob. Optim., 61 (2015), 61: 553. 